\title{
The Unknown Computer Viruses Detection Based on Similarity
}

\author{
Zhongda LIU $^{\dagger a)}$, Nonmember, Naoshi NAKAYA ${ }^{\dagger}$, and Yuuji KOUI' ${ }^{\dagger}$, Members
}

SUMMARY New computer viruses are continually being generated and they cause damage all over the world. In general, current anti-virus software detects viruses by matching a pattern based on the signature; thus, unknown viruses without any signature cannot be detected. Although there are some static analysis technologies that do not depend on signatures, virus writers often use code obfuscation techniques, which make it difficult to execute a code analysis. As is generally known, unknown viruses and known viruses share a common feature. In this paper we propose a new static analysis technology that can circumvent code obfuscation to extract the common feature and detect unknown viruses based on similarity. The results of evaluation experiments demonstrated that this technique is able to detect unknown viruses without false positives.

key words: computer virus, unknown virus, static analysis technology, similarity

\section{Introduction}

Today's society depends on computer networks, and these networks (including the Internet) are expanding rapidly. Correspondingly, the damage of computer viruses becomes more significant every year. Installing anti-virus software is now acknowledged as common sense. The current commercial anti-virus software detects viruses by pattern matching using the signatures of known viruses [1]. Therefore, unknown viruses cannot be detected because there is no information about them in the signature database. For this reason, anti-virus software vendors must always analyze recently discovered viruses and generate their signatures. As a result, users must repeatedly download the latest signature database. So, a technology that does not depend on signatures to detect unknown viruses is needed.

An unknown virus is a virus that cannot be detected with anti-virus software because it doesn't have any corresponding signature, and generally a newly generated virus is an unknown virus. Unknown viruses can be classified into two types.

\section{- New virus}

There is no similarity at all between it and known viruses.

- Variant

There is a certain similarity between it and known viruses.

A virus tends to release itself multiple times with minor

Manuscript received March 21, 2008.

Manuscript revised August 5, 2008.

The authors are with the Graduate School of Engineering, Iwate University, Morioka-shi, 020-8551 Japan.

a)E-mail: liuzd@mn.cis.iwate-u.ac.jp

DOI: 10.1587/transfun.E92.A.190 changes. This change is referred to as a variant of the virus. Because it is easy to create new variants of a virus, a large amount of virus variants are generated. Variants sent in emails are generally almost the same, and the resulting actions and damages to a PC are similar. Thus, there is a certain similarity not only between a variant and its original virus, but also between variants. According to "Virus Report Status for 2007" [2], more than 90\% of unknown viruses are variants of known viruses. So, we can detect more than $90 \%$ of the unknown viruses, if we can detect the variants.

In this paper, we present an unknown virus detection technology based on similarity. Future unknown viruses (variants) can be detected by comparing their similarity with the extracted common features from known viruses. However, currently it is difficult to do static analyses because viruses writers often use code obfuscation technology. Consequently, we developed a static analysis tool which does not disassemble an executable file. We use the tool to extract the API sequence from the code section of an executable file as the common feature. This technology is a useful supplement for current anti-virus software that cannot detect unknown viruses.

\section{Virus Detection Technology}

The virus detection technologies that are not based on signatures can be classified into the following.

- Dynamic observation

Executes an executable file and looks for abnormal actions in a virtual environment [3], [4]

- Static analysis

Analyzes an executable file without executing it, and searches for malicious codes (or features) [5], [6]

Dynamic observation examines the actions of the executable file in a virtual environment, so a virtual machine emulator [7] and a debugger at the OS level are necessary. However, current viruses can detect the virtual environment and disrupt the debugger with anti-emulator [8] and anti-debugger techniques.

In this paper we use the static analysis technology. With this technology, it is necessary to solve the problem that is called code obfuscation.

\section{Code Obfuscation}

Code obfuscation is making code confusing and harder to 


\begin{tabular}{|l|ll|lc|}
\hline Location & \multicolumn{2}{|l|}{ Output of Disassembler } & \multicolumn{2}{l|}{ Actual Disassembly } \\
\hline 0040A000 & 60 & PUSHAD & 60 & PUSHAD \\
0040A001 & E8 01000000 & CALL 0040A007 & E8 01000000 & CALL 0040A007 \\
0040A006 & E8 83C404E8 & CALL E845648E & E8 & \\
0040A007 & & & 83 C4 04 & ADD ESP,4 \\
0040A00A & & E8 01000000 & CALL 0040A010 \\
0040A00B & 0100 & ADD DWORD PTR DS:[EAX],EAX & & \\
\hline
\end{tabular}

Fig. 1 Junk insertion (Bagle.H).

interpret without changing its original semantics. At first it was used for copyright protection and avoiding discovery from vulnerability attackers. Recently, virus writers have been using it to circumvent anti-virus software.

\subsection{Classification}

The basic criterion for classification is the target of an application. Four groups of code obfuscation have been created [9], [10]:

1. Layout obfuscation Change of variables, functions names on random character strings

2. Data obfuscation Variable division, variable type change, variable lifetime change, etc.

3. Control obfuscation Reorder statements, inline method, etc.

4. Preventive obfuscation Protection from disassembly

\subsection{Preventive Obfuscation}

For a virus writer, preventive obfuscation is the most effective. When it is used, even IDA Pro Disassembler [11] incorrectly disassembles close to $94 \%$ of the instructions and approximately $83 \%$ of the functions in a program [12]. Then, it is impossible to analyze an executable file with the technologies of [5] and [6], which are based on IDA Pro.

Here we introduce specific preventive obfuscation techniques that are often used.

- Junk insertion

"Junk" bytes are inserted at selected locations in the instructions to thwart the disassembler. These junk bytes are normally never executed because they are jumped over [13]. Figure 1 shows the code of a variant of the virus Bagle.H. At location 0040A001, the length of the instruction is 5 bytes. The disassembler assumes that the next instruction is "CALL E845648E." In fact, the next start is at 0040A007(ADD ESP,4), and "E8" at location 0040A006 is a junk byte which throws off the disassembler and hides instructions starting at location 0040A007 and 0040A00A. Since E8 is an opcode for the CALL instruction, it looks like a legitimate instruction. The virus writers often use E8 as a junk byte.

- Branch function

This idea is illustrated in Fig. 2 [12]. We can replace

\author{
Original code \\ aı: jmp bı \\ a2: jmp b2 \\ ... \\ an: jmp bn \\ After using branch function \\ a1: call $f_{\mathrm{b}}$ \\ a2: call $f$ b \\ $\cdots$ \\ an: call $f$ b
}

Fig. 2 Branch functions.

$n$ unconditional branches with a branch function $f_{b}$ in a program. The code for the branch function $f_{b}$ is responsible for determining the target location $b_{i}$ based on the location $a_{i}$ it was called from, then branching to the appropriate $b_{i}(1 \leq i \leq n)$. But a branch function does not behave like a "normal" function; it typically does not return to the instruction following the call instruction, but instead branches to some other location. Virus writers often insert junk bytes to the location following the call instruction to thwart the disassembler.

- Opaque predicate

An unconditional branch can also be replaced by a conditional branch that uses an opaque predicate. At runtime, the conditional branch always goes in one direction using predicates that always evaluate to either true or $f$ alse. The virus writers can insert junk bytes in other directions to throw off the disassembler.

- Jump table spoofing

This technique is referred to in Ref. [12]. The basic idea is to take an unconditional jump to an address 1 and to convert it to an indirect jump table where the address $l$ appears as the $k$ th table entry. The table is indexed by the value of an opaque expression that always evaluates to $k$. However, the bounds check for the table uses a table of size $m>k$, leading the disassembler to believe that the jump table contains $m$ entries. Only one of these $m$ entries contains a real code address. The virus writer can put junk bytes into each of the other entries, thereby confusing the disassembler.

\section{Detection of Unknown Viruses}

An executable file has a portable executable (PE) format [14], and is divided into sections, as shown in Fig. 3. In this paper, we developed a static analysis tool named Machine Code Matching (MCM), described in Sect. 4.2. MCM extracst the API sequence from the code section (text) of 


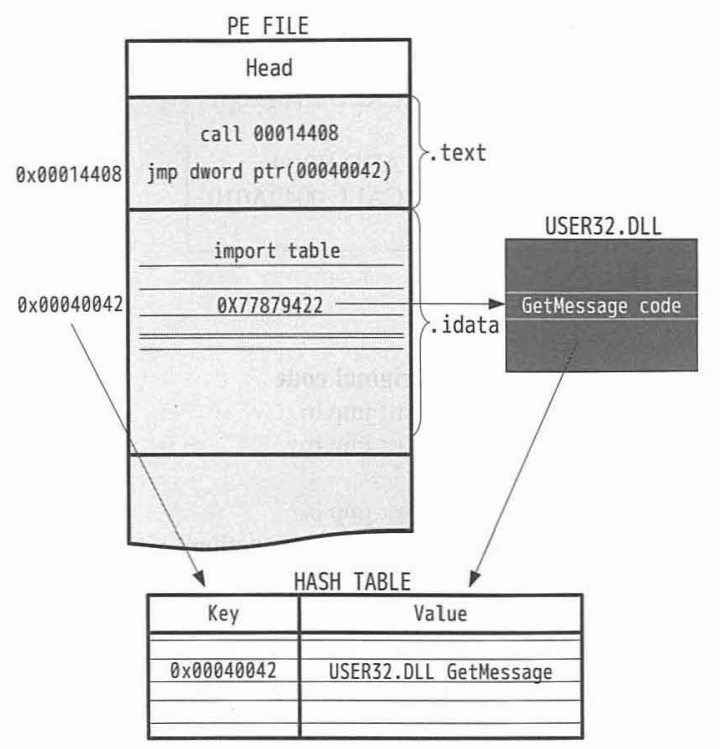

Fig. 3 MCM processing of a PE file.

unpacked known viruses and saves the API sequence in a database as the feature. When a PE file is input, we extract the API sequence from the PE file using MCM, and compare the feature (API sequence) with that of known viruses in the database. If there is a similarity, then we determine the input PE file to be a virus. In order to judge the similarity, we map API sequences to hash sequences, perform global alignment, and measure the similarity with the cosine function and extended Jaccard function. If the output of the similarity is beyond the bounds, it is judged to be similar.

\subsection{API Sequence}

In the Windows OS, DLLs (dynamic link libraries) are Microsoft's implementation of shared libraries and include some functions. The function included in the DLL is called the API function, which is specific for a particular task. All Windows executable files (PE files) use API functions directly or indirectly. In other words, the actions of a PE file can be represented by the API sequence. From this we extract API names in the order of appearance in the code section to make an API sequence, and use it as the feature of the PE file.

\subsection{Machine Code Matching (MCM)}

Normally, to extract an API sequence from a PE file we must disassemble it. But the disassembly takes time and often is intentionally confusing (Sect. 3.2). So, we developed a tool that can extract the API sequence from the code section without disassembly. As mentioned above, we call this tool Machine Code Matching (MCM).

As shown in Fig. 3, when an API function (e.g., GetMessage in USER32.DLL) is called in a PE file, the CALL instruction given by the compiler doesn't transfer control directly to the function in the DLL. Instead, the
Table 1 API sequence and hash sequence.

\begin{tabular}{lc}
\hline API sequence & Hash sequence \\
\hline USER32.dll FindWindowA & 8 \\
USER32.dll EnumWindows & 7 \\
USER32.dll SendMessageA & 9 \\
ADVAPI32.dll RegOpenKeyExA & 3 \\
ADVAPI32.dll RegQueryValueExA & 4 \\
USER32.dll SendMessageA & 9 \\
\hline
\end{tabular}

CALL instruction transfers control to an instruction (JMP DWORD PTR [XXXXXXXX]) that is also in the text section. The JMP instruction looks up an address in the idata (import section), which contains the real pointer of the API function [15]. MCM uses the address as a key, and the name of the API and DLL as a value to create a hash table. With the hash table, we can change API addresses with API names from the start to the end of the code section in the PE file. Then, we obtain a sequence of API names that appear in order, that is, the API sequence of the PE file. Specifically, MCM is implemented by the following procedure.

\section{Hash table creation}

The .idata section (or import table) begins with the array of IMAGE_IMPORT_DESCRIPTORs [15]. Then, according to the array, MCM makes the hash table by using the address that contains the pointer of the API as a key and the name of the API and DLL as a value.

2. Instruction matching

An assembly language is a symbolic representation of the machine code to help a person understand it more easily. Machine code can be one-to-one mapped with the assembly instruction. For example, in the X86 architecture, the assembly instruction of the machine code "B0 61" is "MOV AL." MCM sequentially matches the machine code corresponding to the JMP and CALL instructions (JMP in Fig. 3) from the start to the end of the code section.

3. Validity confirmation and API sequence extraction If MCM matches a machine code, it gets the next four bytes (00040042 in Fig. 3) and then confirms whether the address exists in the hash table. If it exists, MCM inserts the name of the API and DLL corresponding to the key in the hash table into the API sequence; otherwise (for example, if the address is junk), MCM matches the next machine code. By repeating this procedure, MCM can extract the API sequence.

As shown in the example, MCM confirmed the validity of the matched address, and even though it matched the JMP and CALL instruction of the preventive obfuscation, it ignored the next junk bytes. Therefore, preventive obfuscation cannot thwart MCM. By comparison with the disassembler, MCM can dependably extract the API sequence. Table 1 lists part of an API sequence extracted from a normal PE file. The hash values shown in the table are used as examples. In this example, the hash values are large enough to avoid collision, and so the hash sequence is used instead of the API sequence after the API is arranged. 


\subsection{Global Alignment}

In order to compute similarity, first we apply sequence alignment to the hash sequences. Computational approaches to sequence alignment generally fall into two categories: local alignment and global alignment (Fig. 4). Local alignment, which uses the general alignment technique called the Smith-Waterman algorithm [16], is most useful for identifying the similar parts of sequences. Global alignment, which aligns every element in every sequence, attempts to find the best possible alignment from the start to the end of sequences. We selected global alignment and applied the Needleman-Wunsch algorithm [17], which is a general global alignment, to the hash sequences.

The Needleman-Wunsch algorithm is implemented as follows.

1. Use sequence $X=\{x(1), x(2), x(3), \ldots x(m)\}$ and sequence $Y=\{y(1), y(2), y(3), \ldots y(n)\}$ to create a matrix table (see Fig. 5) according to the following expression.

$$
\begin{aligned}
& F(0,0)=0, \\
& F(i, j)=\max \left\{\begin{array}{c}
F(i-1, j-1) \\
+s(x(i), y(i)) \\
F(i-1, j)-1 \\
F(i, j-1)-1 .
\end{array}\right.
\end{aligned}
$$

The score function $s$ is

$$
\begin{cases}s(a, b)=2 & (a=b) \\ s(a, b)=0 & (a \neq b) .\end{cases}
$$

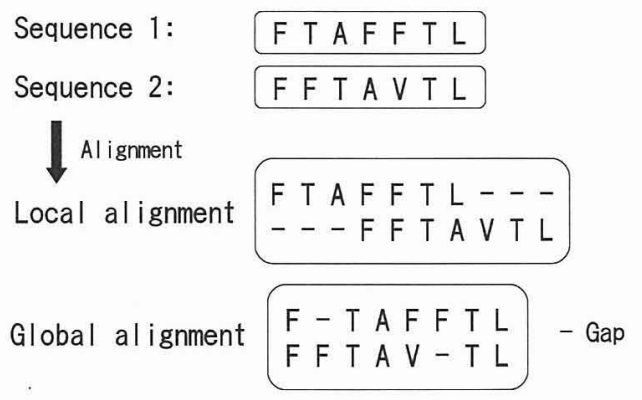

Fig. 4 Local alignment and global alignment.
2. Trace back from the lower right element and connect the values with a line.

3. According to the line formed, insert a gap.

- Line -, insert a gap into the sequence $Y$

- Line I, insert a gap into the sequence $X$

- Line $\backslash$, insert nothing

Figure 6 shows an example using the sequence in Table 1 and a similar sequence.

$$
\begin{aligned}
& X=\{8,7,9,3,4,9\} \\
& Y=\{8,7,3,4,9,6\}
\end{aligned}
$$

Global alignment shows the following. 879349$87-3496$

\subsection{Similarity Measures}

There exist many similarity measures for sequences. For greater efficiency, we chose two popular measures: cosine measure and extended Jaccard measure.

- Cosine measure [18]

$$
S_{\text {Cosine }}(X, Y)=\frac{X^{T} Y}{\sqrt{X^{T} X Y^{T} Y}}
$$

- Extended Jaccard measure [19]

$$
S_{\text {Jaccard }}(X, Y)=\frac{X^{T} Y}{X^{T} X+Y^{T} Y-X^{T} Y}
$$

The cosine measure computes the angle between two sequences and captures a scale invariant according to the similarity. The extended Jaccard measure is computed as the ratio of the number of shared attributes of X AND Y to the number of X OR Y. In this paper the similarity of two sequences is computed as

$$
\frac{S_{\text {Cosine }}(X, Y)+S_{\text {Jaccard }}(X, Y)}{2} .
$$

\begin{tabular}{|c|c|c|c|c|c|c|c|}
\hline & & 8 & 7 & 9 & 3 & 4 & 9 \\
\hline & 0 & -1 & -2 & -3 & -4 & -5 & -6 \\
\hline 8 & -1 & 2 & 1 & 0 & -1 & -2 & -3 \\
\hline 7 & -2 & 1 & 4 & 3 & 2 & 1 & 0 \\
\hline 3 & -3 & 0 & 3 & 4 & 5 & 4 & 3 \\
\hline 4 & -4 & -1 & 2 & 3 & 4 & 7 & 6 \\
\hline 9 & -5 & -2 & 1 & 4 & 3 & 6 & 9 \\
\hline 6 & -6 & -3 & 0 & 3 & 4 & 5 & 8 \\
\hline
\end{tabular}

Fig. 6 Global alignment of two sequences. 


\section{Experimental Evaluation}

We evaluated our technology against real virus variants. In the first experiment, the bound is determined, and we judged the existence of the similarity according to this bound. In the next experiment, we confirmed the detection rate and the false positive rate.

\subsection{Experimental Data}

- Non-viruses

We used 4370 typical but harmless PE files. All of them were collected from common personal computers. There were 844 EXE files and 3886 DLL files, including Microsoft Windows system files, Microsoft development tools, Microsoft Office, and other general applications. We used MCM to extract the API sequences from them. The average size of an API sequence was 414 .

- Variants

We used five variants of Mydoom (A, F, L, M, and O), eight variants of Bagle (J, K, N, O, Y, Z, AB and AE), and eight variants of Warezov $(\mathrm{C}, \mathrm{Gen}, \mathrm{K}, \mathrm{L}, \mathrm{N}, \mathrm{O}$, Q, and AA). All of these are email worms which were filtered at the Iwate University Super Computing and Information Sciences Center. Each worm has many variants. We unpacked them by executing them and dumping them from memory.

\subsection{Bound Determination}

We measured the similarity between the variants of Mydoom, and the similarity between the variants of Mydoom and non-viruses. The results are shown in Fig. 7 and Fig. $8^{\dagger}$. It is known that the similarity between variants of Mydoom is more than 0.5 , and the similarity between variants of Mydoom and non-viruses is less than 0.3. Both Mydoom and non-viruses were correctly identified. Therefore, to decrease false detection as much as possible, the similarity bound was determined to be 0.5 . Therefore, if the similarity between a $\mathrm{PE}$ file and a variant virus exceeds 0.5 , we decide that the $P E$ file is a virus.

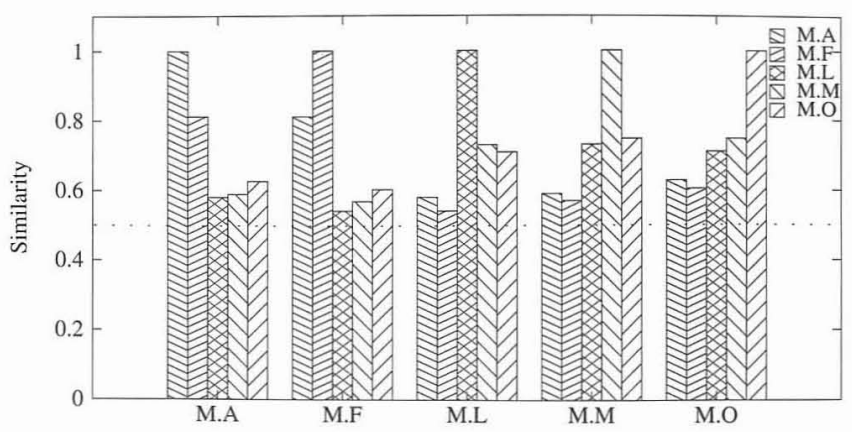

Fig. 7 Similarity between the variants of Mydoom.

\subsection{Variants Detection}

We extracted the features from variants of Bagle and variants of Warezov and conducted a detection experiment. The results are shown in Table 2 and Table 3 ( $\checkmark$ indicates the variant was detected). According to each feature, both tables show whether a variant was detected as the variant when it had been input and whether a non-virus was detected as the non-virus when it has been input. In addition, the layout of variants in each table is the generation order.

In this experiment first it can be seen that the unknown virus that will be generated in the future is able to be detected. For example, using the feature of Bagle.J, we are able to detect Bagle.J, Bagle.K, Bagle.N, Bagle.O, Bagle.Y, Bagle.Z, Bagle.AB, and Bagle.AE (see Table 2 and Fig. 9). The result shows that, if we get a virus, we are able to detect the unknown variants of the virus that will be generated in the future.

Second, we also measured the false positive rate using

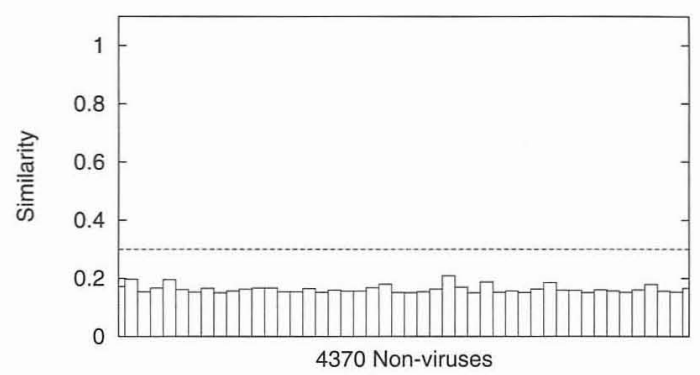

Fig. 8 Similarity between the non-viruses and variants of Mydoom.

Table 2 Detection of the Bagle variants.

\begin{tabular}{l|ccccc}
\hline \hline \multirow{2}{*}{ Feature } & \multicolumn{5}{|c}{ Input } \\
\cline { 2 - 5 } & J & K N O X Y & X AB AE Non-virus \\
\hline Bagle.J & $\checkmark \checkmark \checkmark \checkmark \checkmark \checkmark \checkmark \checkmark$ & $\checkmark$ & $\checkmark$ & $\times$ \\
\hline Bagle.K & $\checkmark \checkmark \checkmark \checkmark \checkmark \checkmark \checkmark \checkmark \checkmark$ & $\checkmark$ & $\checkmark$ & $\times$ \\
\hline Bagle.N & $\checkmark \checkmark \checkmark \checkmark \checkmark \checkmark \checkmark$ & $\checkmark$ & $\checkmark$ & $\times$ \\
\hline Bagle.O & $\checkmark \checkmark \checkmark \checkmark \checkmark \checkmark \checkmark \checkmark$ & $\checkmark$ & $\times$ \\
\hline Bagle.W & $\checkmark \checkmark \checkmark \checkmark \checkmark \checkmark \checkmark$ & $\checkmark$ & $\checkmark$ & $\times$ \\
\hline Bagle.Y & $\checkmark \checkmark \checkmark \checkmark \checkmark \checkmark \checkmark$ & $\checkmark$ & $\checkmark$ & $\times$ \\
\hline Bagle.Z & $\checkmark \checkmark \checkmark \checkmark \checkmark \checkmark \checkmark$ & $\checkmark$ & $\times$ & $\times$ \\
\hline Bagle.AB & $\checkmark \checkmark \checkmark \checkmark \checkmark \checkmark \checkmark$ & $\checkmark$ & $\checkmark$ & $\times$ \\
\hline Bagle.AE & $\checkmark \checkmark \checkmark \checkmark \checkmark \checkmark \checkmark \checkmark$ & $\checkmark$ & $\checkmark$ & $\times$ \\
\hline
\end{tabular}

Table 3 Detection of the Warezov variants.

\begin{tabular}{|c|c|c|c|}
\hline \multirow[b]{2}{*}{ Feature } & \multicolumn{3}{|c|}{ Input } \\
\hline & C Gen K L N O & Q AA & Non-virus \\
\hline Warezov.C & $\checkmark$ & $\checkmark$ & $x$ \\
\hline Warezov.Gen & $\begin{array}{lll}\checkmark & \checkmark \checkmark \checkmark \checkmark\end{array}$ & $\checkmark$ & $x$ \\
\hline Warezov.K & $\checkmark \quad \checkmark \checkmark \checkmark \checkmark \checkmark$ & $\checkmark$ & $x$ \\
\hline Warezov.L & $\begin{array}{llll}\checkmark & \checkmark & \checkmark & \checkmark\end{array}$ & $\checkmark$ & $x$ \\
\hline Warezov.N & $\checkmark \quad \checkmark \checkmark \checkmark \checkmark \checkmark$ & $\checkmark$ & $x$ \\
\hline Warezov.O & $\checkmark \quad \checkmark \checkmark \checkmark \checkmark \checkmark$ & $\checkmark$ & $x$ \\
\hline Warezov.Q & $\checkmark$ & $\checkmark$ & $x$ \\
\hline Warezov.AA & $\begin{array}{lll}\checkmark & \checkmark \checkmark \checkmark \checkmark\end{array}$ & $\checkmark$ & $x$ \\
\hline
\end{tabular}

In Fig. 8 values less than 0.15 were omitted. 


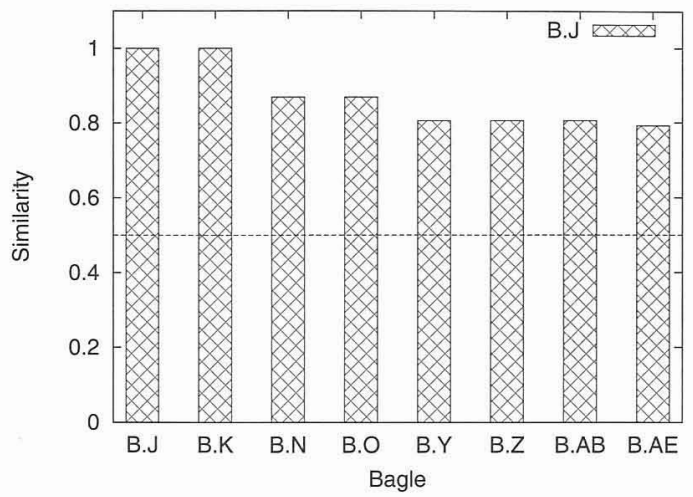

Fig. 9 Detection of the Bagle.J variant.

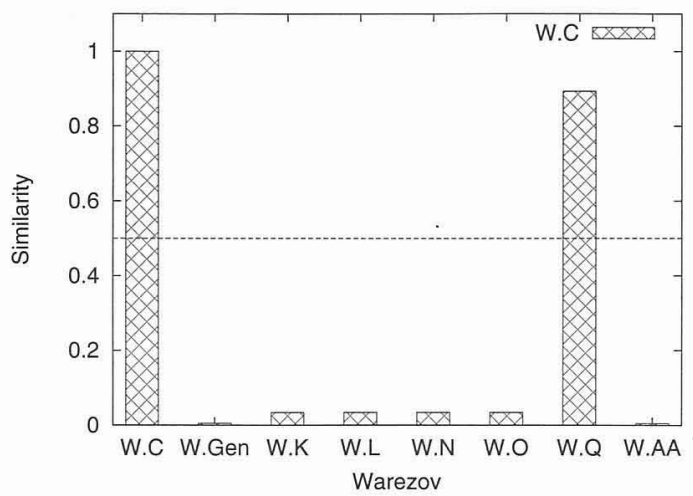

Fig. 10 Detection of the Warezov.C variant.

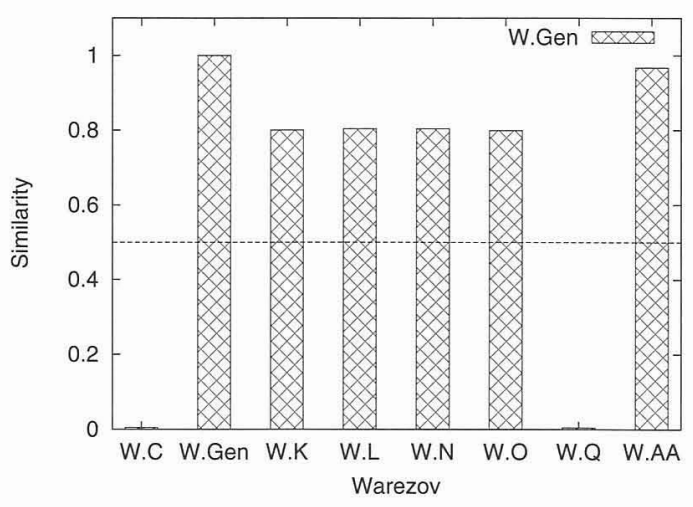

Fig. 11 Detection of the Warezov.Gen variant.

4370 non-viruses. In Table 2 and Table 3, all the results for the non-virus input are indicated by $\times$, which means nonviruses were identified. We also detected 12 other viruses to evaluate the false positive rate, and the maximum similarity between these viruses and non-viruses was 0.42 , less than the bound. All the results show that the false positive rate was $0 \%$.

Last, in Table 3, Fig. 10 and Fig. 11, we found that by using the feature of Warezov.C or Warezov.Q, we cannot detect other variants of Warezov. Also, Warezov.C and Warezov.Q cannot be detected using the features of the other variants. The reason is that Warezov.C and Warezov.Q used not only the DLLs of Windows, but also the DLL prepared by each variant [20], neither of which was used by the other variants. In other words, Warezov.C and Warezov.Q used their own DLL to conceal the API sequence that is the feature of virus Warezov. However, viruses such as Warezov.C and Warezov.Q, are in the minority of existing viruses.

\section{Load Calculation}

Dynamic observation technology is very helpful in understanding the functions of an executable file. The procedure for dynamic observation is as follows.

- Initialize the virtual environment

- Run the executable file

- Monitor the system

(Monitor file changes, registry changes, processes, threads, networks, etc.)

- Recover the virtual environment

This procedure needs a massive amount of time. So, it is well known that dynamic observation technology costs are more than those of static analysis technology.

The conventional static analysis technology is based on the disassembler, and it analyzes disassembled code of an executable file to detect if it is a virus. But, it also takes time to disassemble an executable file. We found that a disassembler needed more than 5 hours to disassemble the 4730 non-viruses (Sect. 5.1), whereas MCM needed only 41 minutes to extract the API sequences from them. Also, as we described in Sect. 3, it is difficult to disassemble a virus correctly. We believe our technology can improve the efficiency of conventional technologies.

\section{Conclusion}

In this paper, we presented a new static analysis technology, that can circumvent code obfuscation to extract the common features of known viruses, and can use the common features to detect unknown viruses. Experimental evaluations demonstrated that our algorithm is able to detect all variants of Mydoom, Bagle, and Warezov, with 0\% false positives.

In the future we will address the problem of DLLs created by viruses such as Warezov.C and Warezov.Q. We also plan to optimize our algorithm to reduce the detection time.

\section{References}

[1] J.O. Kephart and W.C. Arnold, "Automatic extraction of computer virus signatures," Proc. 4th Virus Bulletin International Conference, pp.178-184, Abingdon, England, 1994.

[2] IPA, "Virus report status for 2007," http://www.ipa.go.jp/security/ english/virus/press/200712/virus2007.html

[3] J.R. Crandall, G. Wassermann, D.A.S. de Oliveira, Z. Su, S.F Wu, and F.T. Chong, "Temporal search: Detecting hidden malware timebombs with virtual machines," SIGPLAN Not., vol.41, no.11, pp. $25-36,2006$.

[4] A. MORI, "Detecting unknown computer viruses-A new approach," ISSS, 2003. 
[5] A.H. Sung, J. Xu, P. Chavez, and S. Mukkamala, "Static analyzer of vicious executables (save)," ACSAC'04: Proc. 20th Annual Computer Security Applications Conference, pp.326-334, 2004.

[6] M. Christodorescu and S. Jha, "Static analysis of executables to detect malicious patterns," Proc. 12th USENIX Security Symposium, pp.169-186, Aug. 2003.

[7] VMware, "Vmware," http://www.vmware.com/

[8] P. Ferrie, "Attacks on virtual machine emulators," AVAR, 2006 http://www.symantec.com/avcenter/reference/Virtual_Machine_Threats.pdf

[9] G. Wroblewski, General method of program code obfuscation, Ph.D. Thesis, Wroclaw University of Technology, Institute of Engineering Cybernetics, 2002.

[10] C. Collberg, C. Thomborson, and D. Low, "A taxonomy of obfuscating transformations," Technical Report 148, Department of Computer Science, University of Auckland, July 1997.

[11] DataRescue, "Ida pro," http://www.datarescue.com/idabase/

[12] C. Linn and S. Debray, "Obfuscation of executable code to improve resistance to static disassembly," CCS'03: Proc. 10th ACM Conference on Computer and Communications Security, pp.290-299, New York, NY, USA, 2003.

[13] A. Kapoor, An approach towards disassembly of malicious binary executables, Master's Thesis, The Center for Advanced Computer Studies, University of Louisiana at Lafayette, Nov. 2004.

[14] Microsoft Corporation, Microsoft Portable Executable and Common Object File Format Specification, 2006.

[15] M. Pietrek, Peering Inside the PE: A Tour of the Win32 Portable Execuble File Format. Microsoft Corporation. http://msdn2.microsoft. com/en-us/library/ms809762.aspx

[16] T.F. Smith and M.S. Waterman, "Identification of common molecular subsequences," Journal of Molecular Biology, vol.147, pp.195197, 1981.

[17] S.B. Needleman and C.D. Wuksch, "A general method applicable to the search for similarities in the amino acid sequences of two proteins," J. Molecular Biology, vol.48, pp.443-453, 1970.

[18] I.S. Dhillon and D.S. Modha, "Concept decompositions for large sparse text data using clustering," Mach. Learn., vol.42, no.1/2, pp.143-175, 2001.

[19] A. Strehl and J. Ghosh, "Value-based customer grouping from large retail data-sets," Proc. SPIE Conference on Data Mining and Knowledge Discovery: Theory, Tools, and Technology II, pp.33-42, Orlando, Florida, USA, April 2000.

[20] F-Secure, "F-secure malware information pages: Warezov.c," http://www.f-secure.com/v-descs/warezov_c.shtml

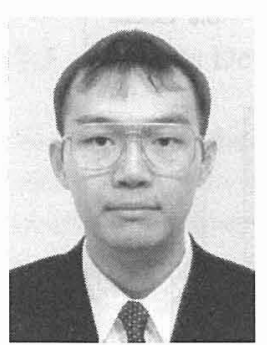

Naoshi Nakaya received his B.Eng., M.Eng. and Ph.D. degrees from Saitama University in 1994, 1996 and 1999, respectively. He is a research associate in the Department of Computer and Information Science $\mathrm{s}$ at Iwate University and working on the research of network systems, network security and evolutionary algorithm.

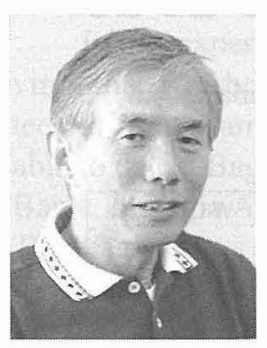

Yuuji Koui graduated from the Science University of Tokyo. He joined Mitsubishi Electric Corporation in 1970, and engaged in the design and development of communicathion systems. He worked on Audio-Visual Information Technology Department in Information Technology R\&D Center of Mitsubishi Electric Corporation. He received the Ph.D. degree in telecommunication engineering from Tohoku University in 1998. He is a professor in the Faculty of Engineering and Graduate School of Engineering Iwate University and working on the research of network system and network security. He is a member of IEEE and the Information Processing Society of Japan.

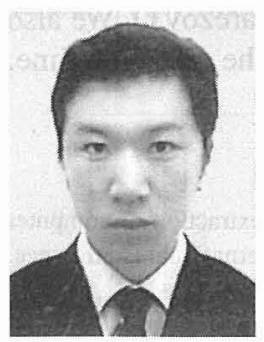

Zhongda Liu received the B.Eng. degree from Jianghan Petroleum University of China in 2000, and the M.Eng. degree from Graduate School of Engineering, Iwate University of Japan in 2007. He is currently pursuing his $\mathrm{Ph} . \mathrm{D}$. degree at Iwate University. His research interests include computer security and network security. 